\title{
Trapping of minority carriers in multicrystalline silicon
}

\author{
D. Macdonalda) and A. Cuevas \\ Department of Engineering, Australian National University, Canberra ACT 0200 Australia
}

(Received 9 October 1998; accepted for publication 28 January 1999)

\begin{abstract}
Abnormally high effective carrier lifetimes have been observed in multicrystalline silicon wafers using both transient and steady-state photoconductance techniques. A simple model based on the presence of trapping centers explains this phenomenon both qualitatively and quantitatively. By fitting this model to experimental data acquired with a quasi-steady-state photoconductance technique, it is possible to determine the trap density, trap energy, and the ratio between the mean-trapping time and mean-escape time. A correlation between trap density and dislocation density in the material has been found. (C) 1999 American Institute of Physics.
\end{abstract}

[S0003-6951(99)03212-X]

Photoconductance measurements of cast multicrystalline silicon (mc-Si) wafers often show unusual behavior, particularly at low illumination levels. The photoconductance decay after a light pulse does not follow a pure exponential function, ${ }^{1,2}$ implying effective lifetimes that increase markedly as the excess carrier density decreases. These apparent lifetimes can reach values in the hundreds of microseconds, even in poor-quality material. Similar problems have been observed when using the quasi-steady-state photoconductance (QSSPC) technique with mc-Si. ${ }^{3,4}$ They have also been reported in other materials, including single-crystal silicon, ${ }^{5}$ thin-film polycrystalline silicon, and CdTe. ${ }^{6}$

These extremely high apparent lifetimes in mc-Si have been suspected to be due to trapping effects, although the physical mechanisms remained unclear and a quantitative explanation of the experimental data had not been attempted. In this letter we examine a trapping model developed by Hornbeck and Haynes, ${ }^{7}$ and also by Fan, ${ }^{8}$ which was used in the mid-1950's to explain unusual carrier behavior in poorquality single-crystal silicon. While the main focus of the Hornbeck and Haynes paper was on the analysis of the transient photoconductance decay, we concentrate here on the steady-state situation, adapting the model for use with the QSSPC technique. In this technique, the photoconductance of the wafers is measured inductively while the sample is subjected to a slowly varying source of illumination. Provided that the rate of change of the light intensity is sufficiently slow, a steady-state analysis can be used to determine the effective lifetime. This technique provides a convenient way of exploring the behavior of the photoconductance under a broad range of illumination conditions.

Figure 1 shows QSSPC data from four mc-Si wafers, depicted on a $\log -\log$ scale. The four samples are from different parts of a $p$-type multicrystalline solar grade silicon ingot grown by directional solidification at Eurosolare, SpA. Wafers labeled 6A, 6B, 6C, and 6D come from the top, near top, center, and bottom of the ingot, respectively. The samples studied were phosphorus gettered, etched, and then given a light phosphorus diffusion and oxidation to passivate the surfaces. Grain sizes were, typically, about $40 \mathrm{~mm}^{2}$ and,

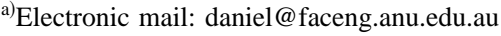

considering that the QSSPC technique measures a region of around $4 \mathrm{~cm}^{2}$, the results are effectively averages over several grains.

The extremely high effective lifetimes at very low injection cause the QSSPC technique to overestimate the lifetimes due to a partial breakdown of the quasi-steady-state assumption. ${ }^{4}$ This can be corrected for by multiplying the lifetimes $\tau_{\text {eff }}$ by a factor $\left(\tau_{\text {light }} 2 \tau_{\text {eff }}\right) / \tau_{\text {light }}$, where $\tau_{\text {light }}$ $52.3 \mathrm{~ms}$, the characteristic decay time of the flash used. To ensure that the correction was adequate, true steady-state measurements were also taken with a low-intensity halogen lamp. These measurements are shown in Fig. 1 and provide extra confidence in the corrected QSSPC measurements.

Figure 1 also shows the adapted trapping model of Hornbeck and Haynes fitted to the QSSPC data. The essential features of the model are illustrated in Fig. 2 in the form of an energy-band diagram. An assumption of the HornbeckHaynes model is that recombination only occurs through a set of deep recombination centers at a rate proportional to $1 / \tau_{r}$, where $\tau_{r}$ is the recombination lifetime, which is assumed constant over the injection-level range considered. The recombination rate perfectly balances the generation

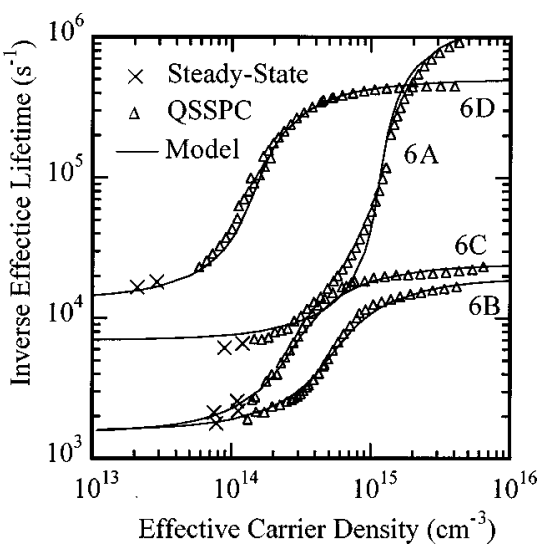

FIG. 1. Quasi-steady-state photoconductance measurements of the effective lifetime for wafers from the top (6A), near top (6B), center (6C), and bottom (6D) of the same mc-Si ingot. True steady-state measurements are indicated by crosses, and the continuous lines represent the trapping model fitted to the data. The values of the fit parameters for each wafer are given in Table I. 


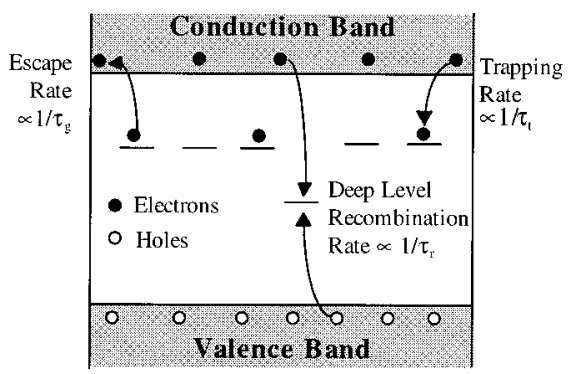

FIG. 2. Energy-band diagram depicting the carrier recombination and trapping processes.

rate, which is provided by steady-state external illumination. Also present are a number of relatively shallow levels, which trap electrons, but do not contribute directly to recombination. The relevant density relating to these traps is the density of normally empty traps $N_{t}$, which is dependent on the temperature and the Fermi level. The material used in this study was $p$-type $\mathrm{mc}-\mathrm{Si}(0.8 \Omega \mathrm{cm})$, and so, the Fermi level is quite close to the valence band, resulting in almost all the traps being empty when in equilibrium at room temperature. Consequently, the density of normally empty traps is effectively equal to the total trap density. Carriers are trapped at a rate proportional to $1 / \tau_{t}$, where $\tau_{t}$ is the mean time before trapping when all the traps are empty. The trapped carriers escape back to the conduction band at a rate proportional to $1 / \tau_{g}$, with $\tau_{g}$ is the mean time spent in a trap, and is independent of the proportion of traps occupied. Under steadystate conditions these rates are in balance.

In order to establish a physical picture of the effect of the traps, first consider the case when the carrier concentration is much greater than the trap density $N_{t}$, a condition that can be obtained with strong illumination. In such a situation the proportion of electrons in the traps is small, even if most of the traps are full, and the excess hole and electron densities, $\Delta p$ and $\Delta n$, respectively, are hence nearly equal. Recombination proceeds through the deep centers at a rate proportional to the photogeneration, reflecting the bulk lifetime of the material. Consider now different experimental conditions with lower light intensities. As the carrier concentration decreases to near and below $N_{t}$, the proportion of electrons in the traps rises. Charge neutrality demands that $\Delta p 5 \Delta n$ $1 n_{t}$, where $n_{t}$ is the trapped electron concentration. Hence, as the trapped electron concentration begins to be appreciable, the excess hole concentration becomes significantly higher than the level that would be expected without traps. The result is a larger photoconductance and, therefore, an increased effective lifetime. The excess conductivity $\Delta \sigma$ (due to the untrapped carriers) is

$$
\Delta \sigma 5 q \Delta n\left(\mu_{n} 1 \mu_{p}\right) 1 q n_{t} \mu_{p},
$$

where $\mu_{n}$ and $\mu_{p}$ are the electron and hole mobilities. At injection levels well below $N_{t}$, the proportion of trapped electrons approaches a constant value determined by the trapping and escape times $\tau_{l}$ and $\tau_{g}$, resulting once again in a constant but significantly larger, effective lifetime.

According to the Hornbeck-Haynes model, the differential equations describing the kinetics of carrier populations in $p$-type material can be written as
TABLE I. Values of the trapping model parameters for the four mc-Si samples shown in Fig. 1. Each sample comes from a different region of the same mc-Si ingot, with a resistivity of $0.8 \Omega \mathrm{cm}$.

\begin{tabular}{lcccc}
\hline \hline Sample & $\begin{array}{c}\text { Bulk lifetime } \\
\tau_{r}(\mu \mathrm{s})\end{array}$ & $\begin{array}{c}\text { Trap density } \\
N_{r}\left(\mathrm{~cm}^{23}\right)\end{array}$ & $\begin{array}{c}\text { Trap/escape } \\
\text { Ratio } \tau_{t} / \tau_{g}\end{array}$ & $\begin{array}{c}\text { Trap energy } \\
E_{C} 2 E_{T}(\mathrm{eV})\end{array}$ \\
\hline 6A (top) & 0.75 & $4.0310^{15}$ & 0.0015 & 0.398 \\
6B & 51 & $1.7310^{15}$ & 0.025 & 0.348 \\
6C (center) & 41 & $9.0310^{14}$ & 0.019 & 0.371 \\
6D (bottom) & 2.0 & $5.1310^{14}$ & 0.008 & 0.409 \\
\hline \hline
\end{tabular}

$$
\begin{aligned}
& \frac{d \Delta n}{d t} 5 g_{e} 2 \frac{\Delta n}{\tau_{r}} 1 \frac{n_{t}}{\tau_{g}} 2 \frac{\Delta n\left(12 n_{t} / N_{t}\right)}{\tau_{t}}, \\
& \frac{d n_{t}}{d t} 52 \frac{n_{t}}{\tau_{g}} 1 \frac{\Delta n\left(12 n_{t} / N_{t}\right)}{\tau_{t}},
\end{aligned}
$$

where $g_{e}$ is the photogeneration rate of electron-hole pairs.

In the steady-state case, the left-hand sides of the above equations are both zero, reducing the relations to

$$
\begin{aligned}
& \Delta n 5 g_{e} \tau_{r}, \\
& n_{t} 5 \frac{N_{t} \Delta n}{\Delta n 1 N_{t} \tau_{t} / \tau_{g}} .
\end{aligned}
$$

Note that for a given photogeneration rate the density of free-electrons $\Delta n$ remains equal to that expected when no traps are present. The main consequence of trapping is an increased number of excess holes, resulting in an enhanced photoconductance.

In the common interpretation of steady or quasi-steadystate photoconductance measurements, the effective carrier lifetime $\tau_{\text {eff }}$ is calculated from the excess conductivity and the photogeneration rate by assuming $\Delta n 5 \Delta p:{ }^{9}$

$$
\tau_{\text {eff }} 5 \frac{\Delta \sigma}{g_{e}\left(\mu_{n} 1 \mu_{p}\right)} .
$$

The effective excess carrier concentration commonly determined in the QSSPC technique is $\Delta n_{\text {eff }} 5 g_{e} \tau_{\text {eff }}$. The model, expressed by Eqs. (1), (4), and (5), in conjunction with Eq. (6), can then be fitted to experimental data by adjusting the parameters $\tau_{r}, \tau_{t} / \tau_{g}$, and $N_{t}$. Note that when $\Delta n$ $@ N_{t} \tau_{t} / \tau_{g}$, then $\tau_{\text {eff }} \rightarrow \tau_{r}$, a constant, as expected (until high injection effects become significant). Also, if $\Delta n$ ! $N_{t} \tau_{t} / \tau_{g}, \tau_{\text {eff }}$ approaches the constant value $\mu_{p} \tau_{r} \tau_{g} /\left[\left(\mu_{n}\right.\right.$ $\left.\left.1 \mu_{p}\right) \tau_{t}\right]$. Our observations indicate that the ratio $\tau_{t} / \tau_{g}$ is, typically, of the order $0.1-0.01$, and so, $\tau_{\text {eff }} \approx 10-100 \tau_{r}$ in this limit, much higher than the true minority-carrier recombination lifetime.

The depth of the trap below the conduction-band $E_{C}$ $2 E_{T}$ can also be estimated via ${ }^{7}$

$$
E_{C} 2 \quad E_{T} 5 k T \log _{e} \bigotimes_{N_{t} \tau_{t}}
$$

where $k$ is Boltzmann's constant and $T 5300 \mathrm{~K} . N_{C}$ is the effective density of states in the conduction band, ${ }^{10}$ with a value of $2.86310^{19} \mathrm{~cm}^{23}$. The calculated trap energies for each of the samples studied are given in Table I, with an average value of $0.3860 .03 \mathrm{eV}$ below the conduction-band edge. It is possible that this value represents an "average" of more than one trap level present in the samples. 


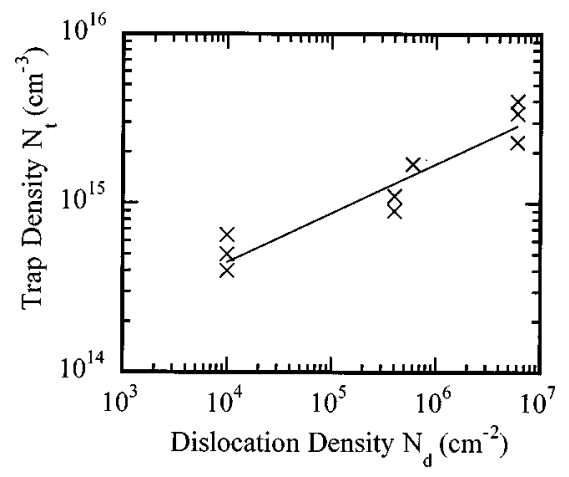

FIG. 3. Dislocation density $N_{d}$ vs trap density $N_{t}$ for samples from the same standard mc-Si ingot. The line of the best fit takes the form $N_{t} 53.1$ $310^{13}\left(N_{d}\right)^{0.29}$.

As Fig. 1 shows, the model is clearly capable of describing the behavior of a variety of mc-Si wafers with very different recombination lifetimes over a broad range of carrier density levels. Table I contains the values of the fit parameters for the curves fitted to QSSPC data from the four wafers studied, indicating that the top of the ingot has the largest trap density and the bottom the lowest.

For the three samples with the highest recombination lifetimes $\tau_{r}$, the model fits the data very well. All these samples have diffusion lengths close to or greater than the sample thickness $(300 \mu \mathrm{m})$. However, for the sample with the lowest lifetime (6A), the diffusion length is only $50 \mu \mathrm{m}$, causing the white-light illumination to produce a nonuniform carrier distribution across the thickness of the wafer. The simplified analysis of the QSSPC data no longer calculates the carrier density correctly, resulting in the calculated lifetimes becoming "stretched" along the horizontal axis. Possible solutions to this problem include the use of thinner wafers, or by illuminating with infrared light, which provides more uniform photogeneration.

Previous work has suggested a link between the presence of dislocations and trapping effects in single-crystal silicon. ${ }^{11,12}$ Figure 3 shows a plot of the trap density against the dislocation density for the samples in Table I, plus data from five additional samples from the same ingot. The dislocation density, which was determined by counting etch pits under an electron microscope, is highest at the top of the ingot, probably due to high thermal stresses resulting from rapid cooling at the end of the casting process.

Figure 3 reveals that a correlation exists between the densities of the trapping centers and dislocations for this particular mc-Si ingot. According to the results in Table I, there also appears to be another relationship between the recombination lifetime $\tau_{r}$ and the ratio $\tau_{t} / \tau_{g}$. Additional work is needed to verify these correlations for other mc-Si materials, identify the physical origin of the trapping centers, and finally, their possible effect on device performance.

In conclusion, we have demonstrated that the anomalously high apparent lifetimes measured in mc-Si at low injection levels are caused by trapping of minority carriers, and that a relatively old theoretical model explains the observed injection-level dependence well. The trapping mechanism affects not only the transient measurements of the photoconductance, but steady-state measurements as well.

M. Stocks and R. Sinton contributed with helpful discussions. Funding was provided by the Australian Research Council. Thanks to Eurosolare SpA for providing the mc-Si wafers.

${ }^{1}$ J. S. Blakemore, Semiconductor Statistics (Pergamon, Oxford, 1962).

${ }^{2}$ R. A. Smith, Semiconductors (Cambridge University Press, Cambridge, 1959).

${ }^{3}$ R. Sinton, A. Cuevas, and M. Stuckings, Proceedings of the 25th IEEE Photovoltaic Specialists Conference, p. 547 (1996).

${ }^{4}$ M. Stocks, Ph.D. thesis, Australian National University, 1998.

${ }^{5}$ A. Romanowski, A. Buczkowski, A. Karoui, and G. Rozgonyi, 8th Workshop on the Role of Impurities and Defects in Silicon Device Processing NREL, p. 196 (1998).

${ }^{6}$ R. K. Ahrenkiel, B. M. Keyes, and D. L. Levi, Proceedings of the 13th European Photovoltaic Solar Energy Conference, p. 914 (1996).

${ }^{7}$ J. A. Hornbeck and J. R. Haynes, Phys. Rev. 97, 311 (1955).

${ }^{8}$ H. Y. Fan, Phys. Rev. 92, 1424 (1953).

${ }^{9}$ R. Sinton and A. Cuevas, Appl. Phys. Lett. 69, 2510 (1996).

${ }^{10}$ M. A. Green, J. Appl. Phys. 67, 2944 (1990).

${ }^{11}$ R. H. Glaenzer and A. G. Jordan, Solid-State Electron. 12, 247 (1969).

${ }^{12}$ K. D. Glinchuk, N. M. Litovchenko, and V. A. Novikova, Sov. Phys. Solid State 8, 777 (1966). 